\title{
Basic MRI for the liver oncologists and surgeons
}

This article was published in the following Dove Press journal: Journal of Hepatocellular Carcinoma

\author{
Lan $\mathrm{N} \mathrm{Vu}^{\prime}$ \\ John N Morelli² \\ Janio Szklaruk' \\ 'Department of Radiology, The \\ University of Texas MD Anderson \\ Cancer Center, Houston, TX, USA; \\ ${ }^{2}$ Department of Radiology, St. John's \\ Medical Center, Tulsa, OK, USA
}

Correspondence: Janio Szklaruk

I5I 5 Holcombe, Blvd., Unit I473,

Houston, TX 77030, USA

$\mathrm{Tel}+\mathrm{I} 7 \mathrm{I} 37453230$

Fax + I 7/3745 I302

Email JSzklaru@mdanderson.org

\begin{abstract}
Magnetic resonance imaging (MRI) is the modality of choice for liver imaging due to its superior contrast resolution in comparison with computer tomography and the ability to provide both morphologic and physiologic information. The physics of MR are complex, and a detailed understanding is not required to appreciate findings on an MRI exam. Here, we attempt to introduce the basic principles of MRI with respect to hepatic imaging focusing on various commonly encountered hepatic diseases. The purpose is to facilitate an appreciation of the various diagnostic capabilities of MR among hepatic oncologists and surgeons and to foster an understanding of when MR studies may be appropriate in the care of their patients.

Keywords: basic MRI liver, HCC, tumor restricted diffusion, Eovist
\end{abstract}

\section{Introduction}

Magnetic resonance imaging (MRI) is the modality of choice for liver imaging due to its superior contrast resolution in comparison with computer tomography (CT) and the ability to provide both morphologic and physiologic information. Unlike CT, MRI does not rely on ionizing radiation. ${ }^{1}$ In distinction to ultrasound, MRI is not operator dependent and has both greater spatial and contrast resolution. By carefully selecting MR parameters, radiologists can noninvasively diagnose both diffuse and focal hepatic disease. In this review, the basic principles of MRI will be explained with respect to hepatic imaging, and a focus on various commonly encountered hepatic diseases will also be given. The purpose is to facilitate an appreciation of the various diagnostic capabilities of MR among hepatic oncologists and surgeons and to foster an understanding of when MR studies may be appropriate in the care of their patients.

\section{Basic MR principles}

The underlying physical principles of MRI are complex. Within each type of sequence, a large number of parameters can be adjusted, each uniquely affecting image quality and contrast. ${ }^{2}$ A precise understanding of each of these parameters is complex. What follows is an attempt to provide a basic-level summary of MRI principles as relevant to abdominal imaging.

Fundamental particles such as protons and electrons have intrinsic properties including mass, charge, and spin. Although many different isotopes can be interrogated with MRI, the abundance of hydrogen-rich tissues such as water, fat, and proteins in the human body renders hydrogen (H1) or proton MRI relatively signal-rich, and thus by far the dominant type of MR performed in clinical practice. When a strong 
magnetic field ( $\mathbf{B}_{\boldsymbol{o}}$, generally in the cranial-caudal direction of the patient) acts upon hydrogen protons, a slight majority of protons align with $\mathbf{B}_{\mathbf{0}}$ in a low-energy parallel fashion. A minority align $180^{\circ}$ opposed in a higher energy, antiparallel state. Thus, the net longitudinal magnetization is aligned with $\mathbf{B}_{\mathbf{0}}$. In addition to aligning parallel or antiparallel to $\mathbf{B}_{\mathbf{o}}$, the protons simultaneously resonate or precess out of phase at a specific frequency called the Larmor frequency, which is proportional to the strength of the field $\mathbf{B}_{\mathbf{0}}$. At this point, because the protons spins are not in phase, there is no transverse magnetization. For water protons at $1.5 \mathrm{~T}$, this is 63.87 MHz.

To obtain an image, a radiofrequency (RF) pulse at the Larmor frequency is applied perpendicular to $\mathbf{B}_{\mathbf{0}}$ in the region of interest. With the absorption of the RF pulse, the number of protons in the higher energy antiparallel state will increase, resulting in a net longitudinal magnetization angled away from $\mathbf{B}_{\mathbf{0}}$ (ie, flip angle). Simultaneously, the protons will begin to precess in phase, resulting in development of a net horizontal magnetization (ie, in the transverse $x-y$ plane). Following absorption of the RF pulse, the protons eventually return to their normal low-energy state (ie, recovery of longitudinal net magnetization) aligned with $\mathbf{B}_{\mathbf{0}}$ and progressively fall out of phase (ie, decay of horizontal magnetization). In this process, RF signals are emitted and detected electronically in order to construct the image. The emitted signal provides the information to form the MR image. This signal has two components. One component, called T1, is due to the release of energy as the net longitudinal magnetization of the protons recovers to align with $\mathbf{B}_{\mathbf{0}}$. T2 is the signal from the decay of horizontal magnetization as phase coherence is lost following RF excitation. T1 signal is tissue specific and also dependent on the surrounding structure or lattice (also known as spinlattice relaxation). A shorter $\mathrm{T} 1$ relaxation time results in higher $\mathrm{T} 1$ signal. $\mathrm{T} 2$ signal, on the other hand, is dependent on the spin-spin interactions between protons and their closest neighbors within tissues and is thus dependent upon the composition of adjacent molecules and surrounding atoms within a molecule. The longer the duration of proton dephasing, the higher the $\mathrm{T} 2$ signal will be. Both $\mathrm{T} 1$ and $\mathrm{T} 2$ signals are emitted simultaneously after the RF pulse is turned off, and different materials and tissues have different intrinsic $\mathrm{T} 1$ and T2 properties. Pathological conditions, importantly, also affect emitted $\mathrm{T} 1$ and $\mathrm{T} 2$ signals.

The main parameters that are modified to generate these images are echo time (TE), repetition time (TR), and flip angle. By selecting appropriate parameters and RF pulses, the resulting signal is modified to yield weighted images. We can then generate T1-weighted images (T1WIs) or T2-weighted images (T2WIs). A T1WI emphasizes T1 signal, and T2WI is predominantly $\mathrm{T} 2$ signal, in tissues. The variation of additional parameters can generate images with short acquisition times (gradient echo) versus a slower acquisition with more robust images (spin-echo). Further descriptions of the physics underlying acquisition of each series are beyond the scope of this manuscript.

A standard MR examination of the liver is composed of seven main series (Figure 1): T1WI (pre-contrast), in-phase, out-of-phase, T2WI, diffusion-weighted images, magnetic resonance cholangiopancreatography $[\mathrm{MRCP}]$ images, and postcontrast $\mathrm{T} 1$ images. This manuscript reviews each pulse sequence used in the standard liver MR examination by providing clinical examples of liver pathology and the contribution of each of the series to the diagnosis.

\section{TI-weighted imaging}

The term T1WI refers to an imaging series that demonstrates low signal for water molecules, ie, dark. This is due to the physical properties of water in a magnetic field. In contrast, materials that may have high intrinsic $\mathrm{T} 1$ signal are T1 bright or hyperintense (to the paraspinal musculature). The dark or bright signals are normally compared to normal musculature signals. Hyperintense material on T1W includes fat, methemoglobin, melanin, proteinaceous fluid, paramagnetic substances (eg, manganese, copper), and chelated gadolinium contrast. Tissues with low T1 signal are $\mathrm{T} 1$ dark or hypointense, including iron, water, air, bone, and collagen (Table 1). T1W images are excellent for delineation of abdominal anatomy. When examining an abdominal T1W image, a normal liver should demonstrate uniform T1 signal similar or isointense to the paraspinal muscles and slightly hyperintense to the spleen. Abnormal signal of the liver parenchyma on T1WI is suggestive of pathology. For example, iron is deposited in the liver in hemochromatosis. As iron is low signal on T1WI, the more iron deposited, the darker the signal of the liver compared to the paraspinal

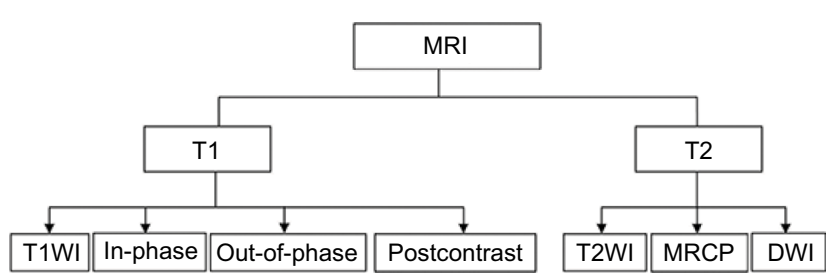

Figure I Basic MR sequences in liver imaging.

Abbreviations: MR, magnetic resonance; MRI, MR imaging; TIWI, TI -weighted image; T2WI, T2-weighted image; MRCP, magnetic resonance cholangiopancreatography; DWI, diffusion-weighted imaging. 
Table I Intrinsic TI and T2 signals of common materials in tissues in MRI

\begin{tabular}{lll}
\hline $\begin{array}{l}\text { MR } \\
\text { signal }\end{array}$ & High signal (bright) & Low signal (dark) \\
\hline TI & Fat, melanin, blood & \\
& (methemoglobin) & Iron \\
& Proteinaceous fluid & Water \\
& Paramagnetic substances & Air \\
& (manganese, copper) & Bone, collagen \\
& Chelated gadolinium contrast & \\
T2 & Water & Air \\
& Edema & Bone \\
& Fat & Chronic blood/hemosiderin \\
& Blood & Acute blood (intracellular \\
& Most tumors & deoxyhemoglobin) \\
& & Early subacute blood \\
& & (methemoglobin)
\end{tabular}

Abbreviations: TIWI, TI-weighted image; T2WI, T2-weighted image.

muscles (Figure 2A and B). Liver masses, both benign and malignant, are also commonly low-signal intensity on T1WI. For example, cysts, hemangiomas, metastases, and primary liver tumors are typically hypointense to the liver on T1WI. The T1WI are not useful to distinguish a benign from a malignant lesion. Melanin has high signal on T1WI, and thus, melanocytic melanoma metastases to the liver may appear as hyperintense masses (Figure 3). Some blood products are also bright on T1WI. This can be useful in identifying hemorrhage within hepatic tumors such as hepatocellular carcinomas (Figure 4C) and adenomas.

\section{In-phase and out-of-phase}

T1WI can be characterized as 1) in-phase and 2) out-ofphase, a distinction referring to the relative signal coming from water and fat within a voxel. When water and fat signals within a voxel are additive, the image is known as "in-phase" image. When water and fat signals in a voxel are in opposite direction and cancel each other, the image is known as "outof-phase image". Out-of-phase images are readily identified by the etching or chemical shift artifact along the periphery of organs containing both fat and water (Figure 5B), such as the liver. These images are used to identify fat in the liver or within a liver lesion. For example, in diffuse hepatic steatosis, the entire liver loses signal intensity on out-of-phase compared to in-phase images (Figure 5). This signal loss is based on the cancellation of water and fat signal within a voxel on out-of-phase images. Out-of-phase images are also useful to identify fat within a lesion, a finding characteristic of both hepatocellular adenomas and adenocarcinomas (Figure 4A and B; Figure $6 \mathrm{~A}$ and $\mathrm{B}){ }^{3-6}$

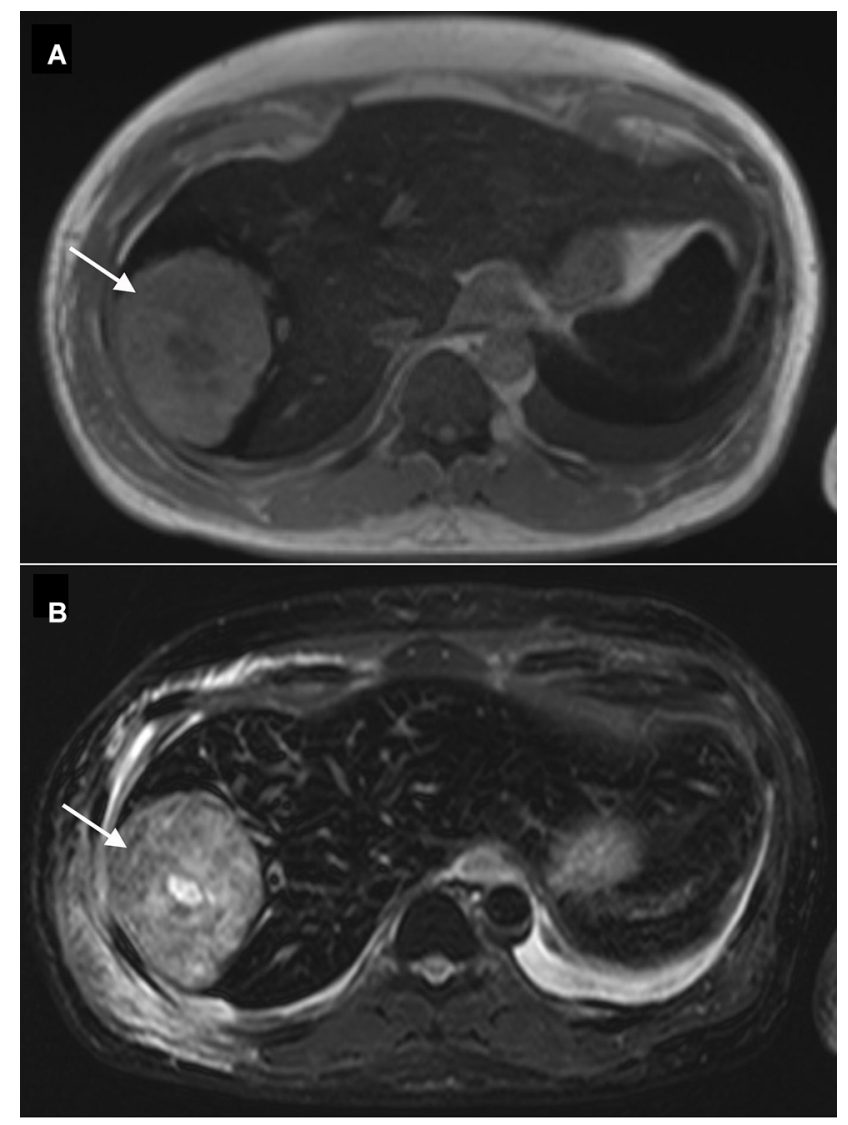

Figure 2 A 60-year-old male with HCC and secondary hemochromatosis from repeated blood transfusions for beta thalassemia minor.

Notes: HCC and secondary hemochromatosis (A) TIW axial image shows both the liver parenchyma and the spleen with diffuse low signal (dark) due to iron deposition secondary to a long prior history of blood transfusions. An isointense TI mass in the right hepatic lobe (arrow) is HCC. (B) T2W-FS axial image shows diffusively low signal of the liver parenchyma and the spleen, consistent with secondary hemochromatosis. The HCC (arrow) has heterogeneous hyperintense T2 signal. Abbreviations: HCC, hepatocellular carcinoma; TIW, TI-weighted; T2W-FS, T2weighted fat suppressed.

It is important to note that if a lesion is $100 \%$ fat, there will be no signal loss between the in-phase and out-of-phase images. This is because there is no cancellation of signal of fat by water. This may be seen in a hepatic lipoma.

\section{T2-weighted imaging}

On routine T2WI, fluid, edema, fat, and some hemorrhagic products are bright (Table 1). Masses such as hepatic cysts, biliary hamartomas, abscesses, and hemangiomas are bright compared to liver on T2WI. T2WI are generally obtained with fat suppression or nulling of fat signal, which increases the contrast between a lesion and the liver. Solid hepatic masses are also typically hyperintense on T2WI but not as bright as cysts (Figure 7). Solid lesions are usually isointense to the spleen. T2WI are excellent for the detection of liver lesions due to high contrast and a small dynamic range. The T2WIs 


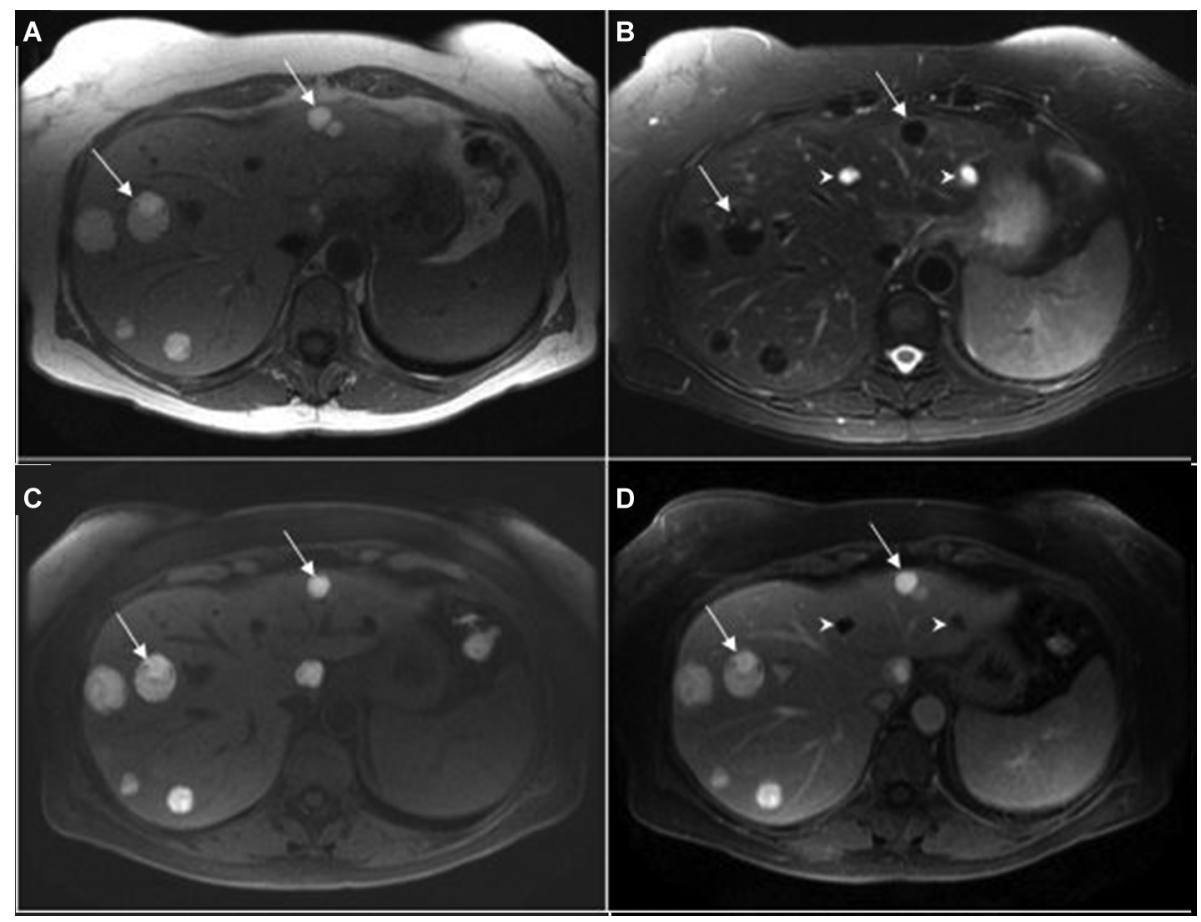

Figure 3 A 61 -year-old female with metastatic melanoma.

Notes: Hepatic metastatic melanoma. (A) TIW; (B) T2W; (C) TIW-FS-precontrast; (D) TIW-FS-postcontrast. The liver is normal in signal on TIW images, isointense to the muscle (A). Hepatic metastatic melanoma in this patient presents as multiple hyperintense TI (A, C, D)/hypointense T2 (B) lesions scattered throughout the liver (arrows). The lesions are bright on TI due to high melanin content. Note that when FS is applied to the TI sequence (C), the liver lesions become even more conspicuous compared to a non-fat-saturated TI (A). For this reason, TI-FS is always obtained prior to gadolinium contrast injection. Incidentally, there are two simple cysts (arrow heads) seen in segments II and IV.

Abbreviations: TIW, TI-weighted; FS, fat saturation; T2W, T2-weighted.

are useful in the characterization of benign lesions such as cysts and hemangiomas (Figure 5C). These masses will maintain their hyper-intensity as the $\mathrm{T} 2$ weighting is increased. By contrast, solid metastases will lose their hyperintensity as the T2 weighting is increased. The increase in T2WI is achieved by increasing the TE and TR. ${ }^{1,2}$

\section{MR cholangiopancreatography}

MRCP is a special type of heavily T2WI. The MR parameters are adjusted to yield an image that results in high contrast between fluid signal and background. Thus, bilious fluid and pancreatic fluid within the biliary tree and pancreas are bright relative to surrounding tissues. Fluid in the duodenum and stomach will also be bright, and this may obscure the visualization of the bile ducts. MRCP is often obtained in patients with suspected pancreaticobiliary disease, particularly in those with comorbidities precluding Endoscopic Retrograde Cholangiopancreatography (ERCP). MRCP can detect alterations in ductal caliber or a signal void within the pancreaticobiliary tree, such as a calculus or mass (Figure 8). The MRCP is the "extreme T2W" image with a very long TE and long TR.

\section{T2*-weighted imaging $(\mathrm{T} 2 * \mathrm{WI})$}

$\mathrm{T} 2 * \mathrm{WI}$ is a derivative of $\mathrm{T} 2 \mathrm{WI}$. This imaging sequence, called gradient echo sequence, does not use $180^{\circ}$ refocusing $\mathrm{RF}$ pulses after an excitation pulse. The $\mathrm{T} 2$ signal that is emitted by the tissue, known as T2*, will be heavily affected by surrounding microscopic and macroscopic materials with high magnetic susceptibility causing local magnetic field inhomogeneity within the tissues. This sequence is sensitive and used to detect calcium, hemorrhage, and iron deposition, which all show low-signal and "blooming artifacts" in the image.

Advanced image techniques using T2*WI beyond the basic gradient echo T2WI include susceptibility-weighted imaging, perfusion MRI, functional MRI, and iron overload imaging. ${ }^{7}$ In susceptibility-weighted imaging, small hemorrhagic lesions (ie, diffuse axonal injury) and small vessels are easily detectable. Perfusion MRI is used to measure blood flow which is useful in grading tumors and determining salvageable tissue in a hyperacute stroke patient. Functional MRI measures the oxygen levels in hemoglobin. Iron overload imaging is used to noninvasively measure the iron concentration within an organ. Of the four techniques, the 

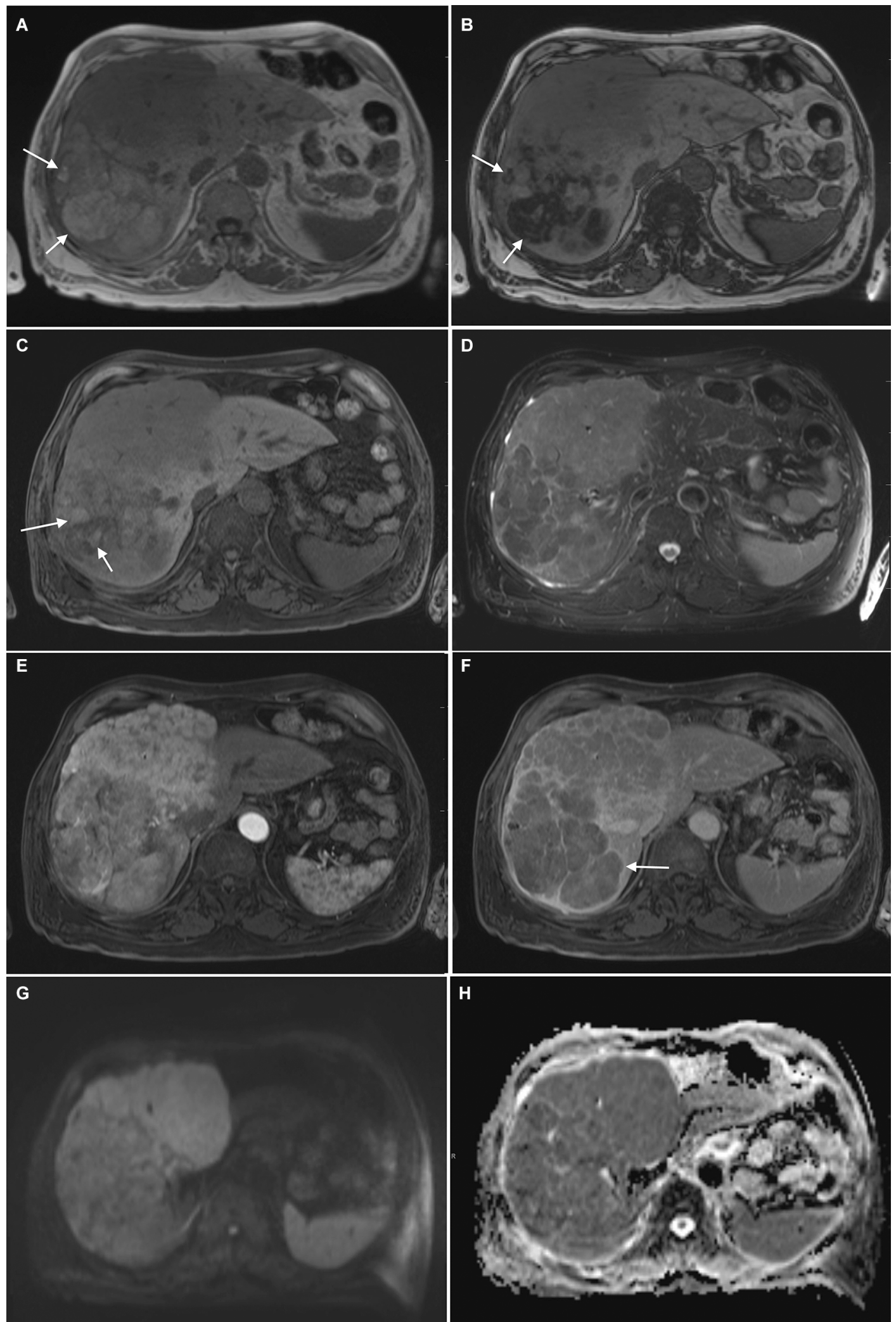

Figure 4 A 68-year-old male with recent diagnosis of HCC.

Notes: HCC. (A) TI-in-phase shows a large conglomerate mass occupying most of the right hepatic lobe. The left hepatic lobe is spared. Arrows show fat content within the HCC which loses signal in the out-of-phase image. (B) TI-out-of-phase shows this fat content (arrows). (C) TI-FS-precontrast shows foci of increased TI signal (arrows) which are indicative of hemorrhage. (D) T2-FS shows the heterogeneous T2 signal of the HCC mass. (E) TI-postcontrast early arterial phase shows intense contrast enhancement. (F) TI-postcontrast portal phase shows that the mass loses enhancement with respect to the liver parenchyma ("washout"). Note the enhancing rim around the HCC (arrow) is defined radiologically as a capsule. (G) DWl shows increased signal within the mass, and the (H) ADC Map shows low signal within the HCC, indicative of restricted diffusion.

Abbreviations: $\mathrm{HCC}$, hepatocellular carcinoma; FS, fat suppression; DWI, diffusion-weighted imaging; ADC, apparent diffusion coefficient. 

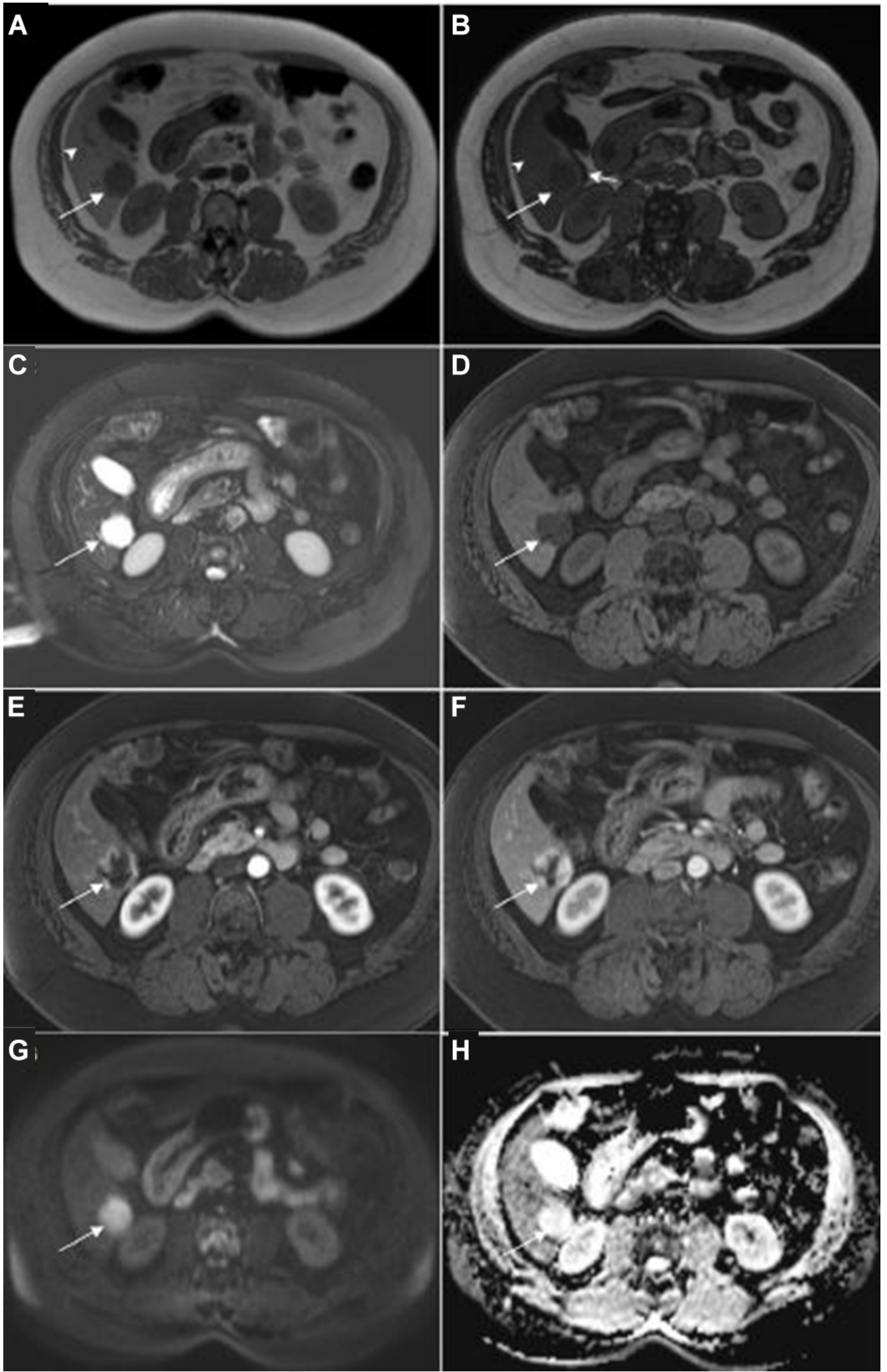

Figure 5 A 65-year-old female with history of papillary thyroid carcinoma.

Notes: Hepatic steatosis and hepatic hemangioma. (A) TI-in-phase shows increased TI signal of the liver (arrow head) compared to the hemangioma (arrow A-H). (B) TI-out-of-phase shows a decrease of signal in the liver (arrow head) compared to the in-phase sequence. The liver is now isointense to the hemangioma. This finding is indicative of a fatty liver. Note the out-of-phase image is readily identified by the etching or chemical shift artifact along the periphery of organs containing both fat and water (medium arrow). (C) T2-FS shows a hemangioma with typical very bright T2 signal. (D) TI-FS-precontrast shows the hemangioma with hypointense TI lesion. (E) TI-postcontrast arterial phase shows peripheral nodular enhancement of the hemangioma. (F) TI-postcontrast delay phase shows a hemangioma with centripetal contrast filled in. Hemangiomas do not demonstrate restricted diffusion as seen in (G) with high DWI signal $(\mathbf{H})$ and low values on the ADC map (arrow).

Abbreviations: $\mathrm{FS}$, fat saturation; DWl, diffusion-weighted imaging; ADC, apparent diffusion coefficient. 

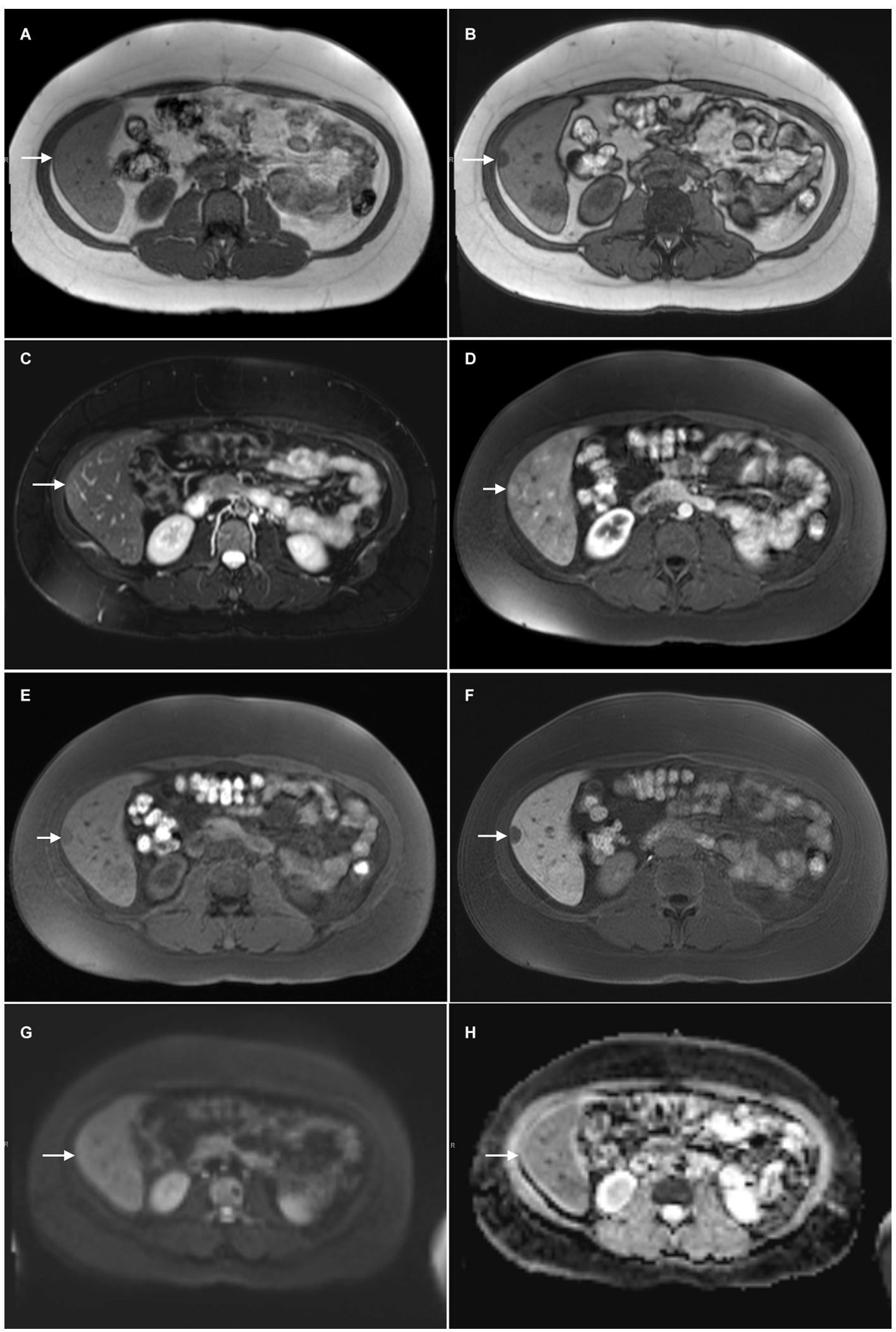

Figure 6 A 39-year-old female without any significant medical history with multiple biopsy-proven hepatic adenomas.

Notes: Hepatic adenoma. (A) TI-in-phase; (B) TI-out-of-phase; (C) T2-FS; (D) TI-postcontrast arterial phase using gadoxetic acid; (E) TI-postcontrast 5-minute delay; (F) TI-postcontrast 20-minute delay; (G) DWl; (H) ADC map. An adenoma in segment VIII (arrow A-H) demonstrates signal loss in the OOP-TI compared to the IPTI. This is in keeping with fat in the lesion (A, B). There is homogeneous enhancement (D) and no retention of gadoxetic acid on the 20-minute delayed images (F). Like FNH, hepatic adenoma is a benign lesion but can restrict diffusion $(\mathbf{G}, \mathbf{H})$.

Abbreviations: T2-FS, T2-weighted-fat suppressed; DWI, diffusion-weighted imaging; ADC, apparent diffusion coefficient; OOP-TI, out-of-phase TI: IPTI, in-phase TI; $\mathrm{FNH}$, focal nodular hyperplasia. 

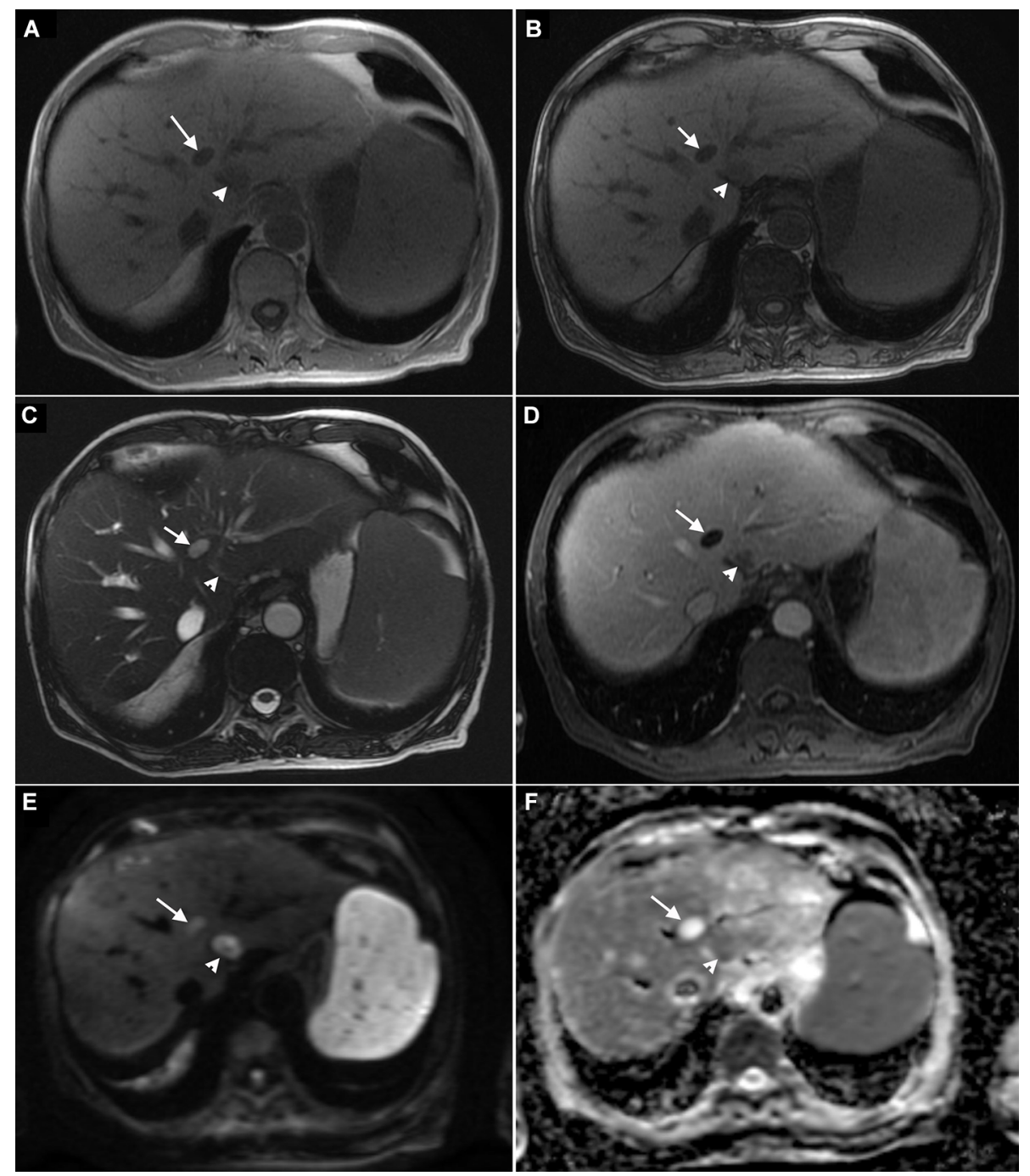

Figure 7 A 64-year-old male with a history of hepatitis C-related cirrhosis, status post liver transplant for HCC.

Notes: Hepatic cyst and HCC. (A) TI-in-phase; (B) TI-out-of-phase; (C) T2-FS; (D) TI-post-contrast delay phase; (E) DWI; (F) ADC map. There is no signal loss in the liver between the IP-TI (A) and OOP-TI (B). This indicates no fat in the liver. The cyst (arrow A-F) in segment IV demonstrates low TI (A, B) and high T2 signal (C) without any contrast enhancement (D). A cyst shows no restricted diffusion with high DWI (E) signal and low values on the ADC map (F). A lesion in segment I of the liver (arrow head A-H) is an HCC. Note that this tumor shows heterogeneous enhancement (D) with washout (not shown) and restricted diffusion (high DWI signal and low values on $A D C$ map).

Abbreviations: HCC, hepatocellular carcinoma; T2-FS, T2-weighted-fat suppressed; DWI, diffusion-weighted imaging; ADC, apparent diffusion coefficient; IP-TI, in-phase TI; OOP-TI, out-of-phase TI.

first three are mainly used in brain imaging. Of the abovementioned techniques, only iron overload imaging is relevant for abdominal imaging.

\section{Diffusion-weighted imaging (DWI)}

DWI is also a subtype of T2WI. The DWI MR images provide information about the Brownian motion of water molecules in a voxel. The images can be acquired with varying degrees of sensitivity to such motion. Background liver has low signal intensity on DWI. In addition, the image parameters can be modified to cancel the signal from bile ducts and vessels. Thus, there is a high level of contrast between hepatic lesions and background liver, making DWI a highly sensitive modality for detection of focal hepatic lesions. Water molecules that freely move within a voxel are termed "unrestricted" in their diffusion and result in low signal intensity on DW images. Water molecules that do not move freely are termed "restricted diffusion" and demonstrate high signal on DWI. The degree of diffusion restriction can be quantified via an apparent diffusion coefficient map (ADC map) constructed from the DWI dataset. Cystic masses and necrosis have high ADC values, nonrestricted diffusion (Table 2). Solid masses and tumors have low ADC values. ${ }^{8}$ ADC maps have been used to evaluate response to treatment. ${ }^{9,10}$ Tumor necrosis following treatment demonstrates increased ADC values, whereas residual or recurrent cellular tumor has low ADC values (Figure 9). 
Table 2 Differentiating tumor and nontumor lesions using DW and ADC mapping signaling

\begin{tabular}{lll}
\hline & High ADC (bright) & Low ADC (dark) \\
\hline High DWI & $\begin{array}{l}\text { Benign lesions (ie, cysts, } \\
\text { adenoma, hemangioma) }\end{array}$ & Tumors/recurrent tumors \\
& Fumor necrosis & Abscesses \\
\hline
\end{tabular}

Abbreviations: DWI, diffusion-weighted imaging; $A D C$, apparent diffusion coefficient; $\mathrm{FNH}$, focal nodular hyperplasia.

\section{Contrast-enhanced images}

As opposed to CT that uses iodinated contrast, contrastenhanced MRI is typically performed using intravenous administration of gadolinium chelate contrast media. The evaluation of liver lesions with contrast-enhanced MRI exploits the fact that many hepatic lesions have a different vascular architecture than the normal hepatic parenchyma. After intravenous injection, T1WIs are sequentially acquired. Paramagnetic gadolinium ions travel through the bloodstream shortening $\mathrm{T} 1$ relaxation time in nearby tissues and thus increasing T1 signal intensity on the final MR image. Different from CT, the volume of contrast is a fraction of that for CT. Only $10 \mathrm{cc}$ of contrast is used in MR compared to over $100 \mathrm{cc}$ on CT.
The first post-Gd phase is the late arterial phase. Late arterial phase imaging is performed at approximately $15-30$ seconds after injection and refers to the images acquired when contrast first opacifies the early the portal veins with contrast (Figure 10C). This is useful in the detection of hypervascular lesions. This is essential in the characterization and detection of hepatocellular carcinoma (HCC). In the portal venous phase, images are obtained at 45-75 seconds following contrast injection. At this point, contrast completely opacifies the portal veins, and the liver parenchyma enhances homogeneously relative to the hepatic arteries. Delayed-phase images are obtained 2 and 5 minutes after contrast injection. At the time of acquisition of delayed-phase images, contrast is being slowly excreted, typically by the kidneys, and thus, the liver begins to enhance less. The portal venous, delayed and late phases provide information on lesion washout or late enhancement. The latter is seen in cholangiocarcinomas and liver metastases.

Contrast-enhanced imaging is a powerful technique to discriminate various hepatic lesions. For example, hepatic cysts have imperceptible walls without enhancement (Figure 7). A cavernous hemangioma, on the other hand,

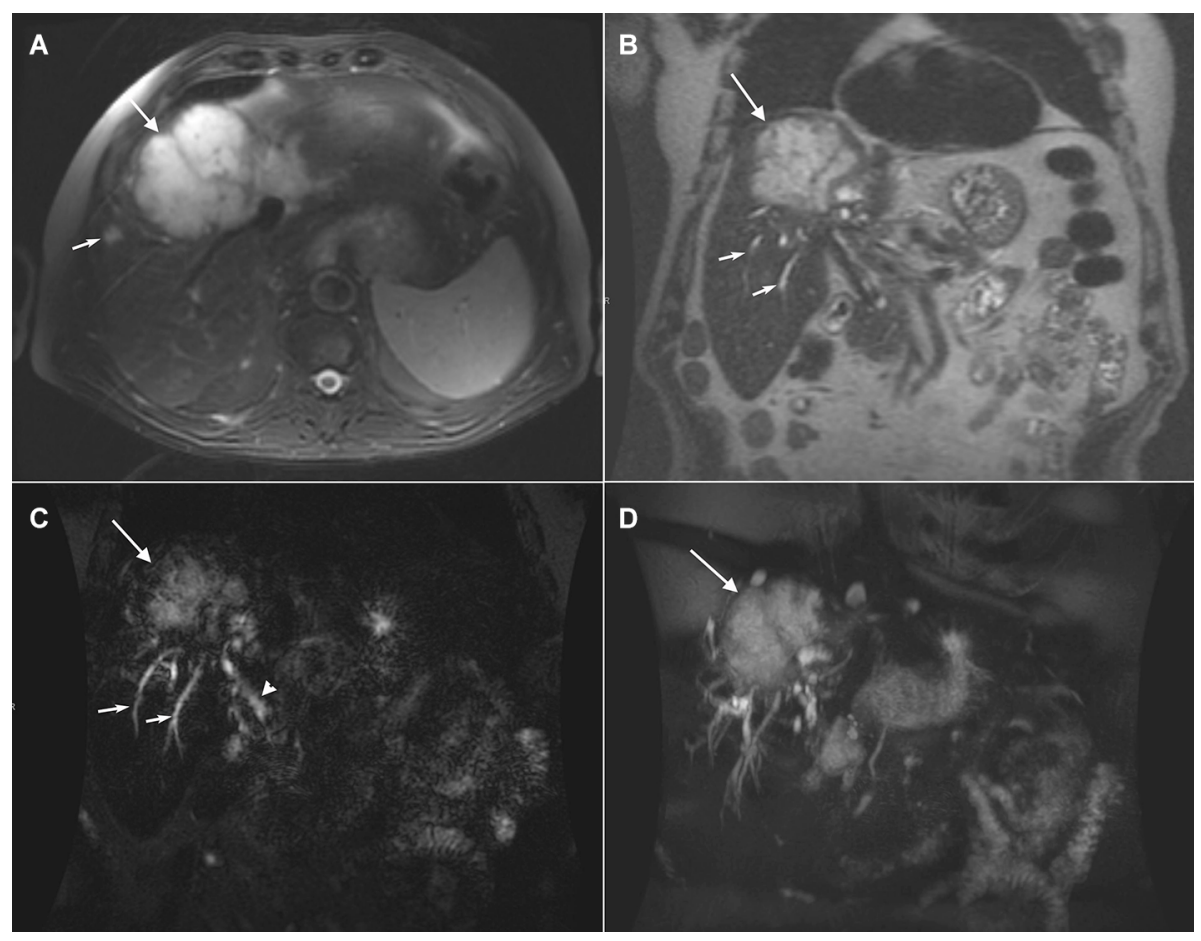

Figure 8 A 66-year-old male with cholangiocarcinoma.

Notes: Cholangiocarcinoma. (A) T2W axial image shows a large hyperintense T2 cholangiocarcinoma (big arrow) in segment VIII. The mass obstructs the biliary confluence and causes intrahepatic biliary duct dilation (small arrow). (B) T2W coronal image shows the same mass (big arrow) in the coronal view with intrahepatic biliary duct dilatation (small arrows). (C) 3D-MRCP technique provides a 3D view of the biliary tree with high contrast between static fluid signal within the biliary tree and background. $M R C P$ examines bile duct dilation, stenosis, and obstructing masses/stones within the biliary tree. Here, the mass (big arrow) is obstructing the biliary tree causing intrahepatic biliary dilation (small arrows). The common bile duct is normal in caliber (arrowhead). (D) A 3D-MRCP reformatted using MIP is used to look at the biliary tree in a threedimensional projection. The arrow is the same mass that was seen in A-C.

Abbreviations: T2W, T2-weighted; 3D-MRCP, three-dimensional magnetic resonance cholangiopancreatography; MIP, maximum intensity projection. 


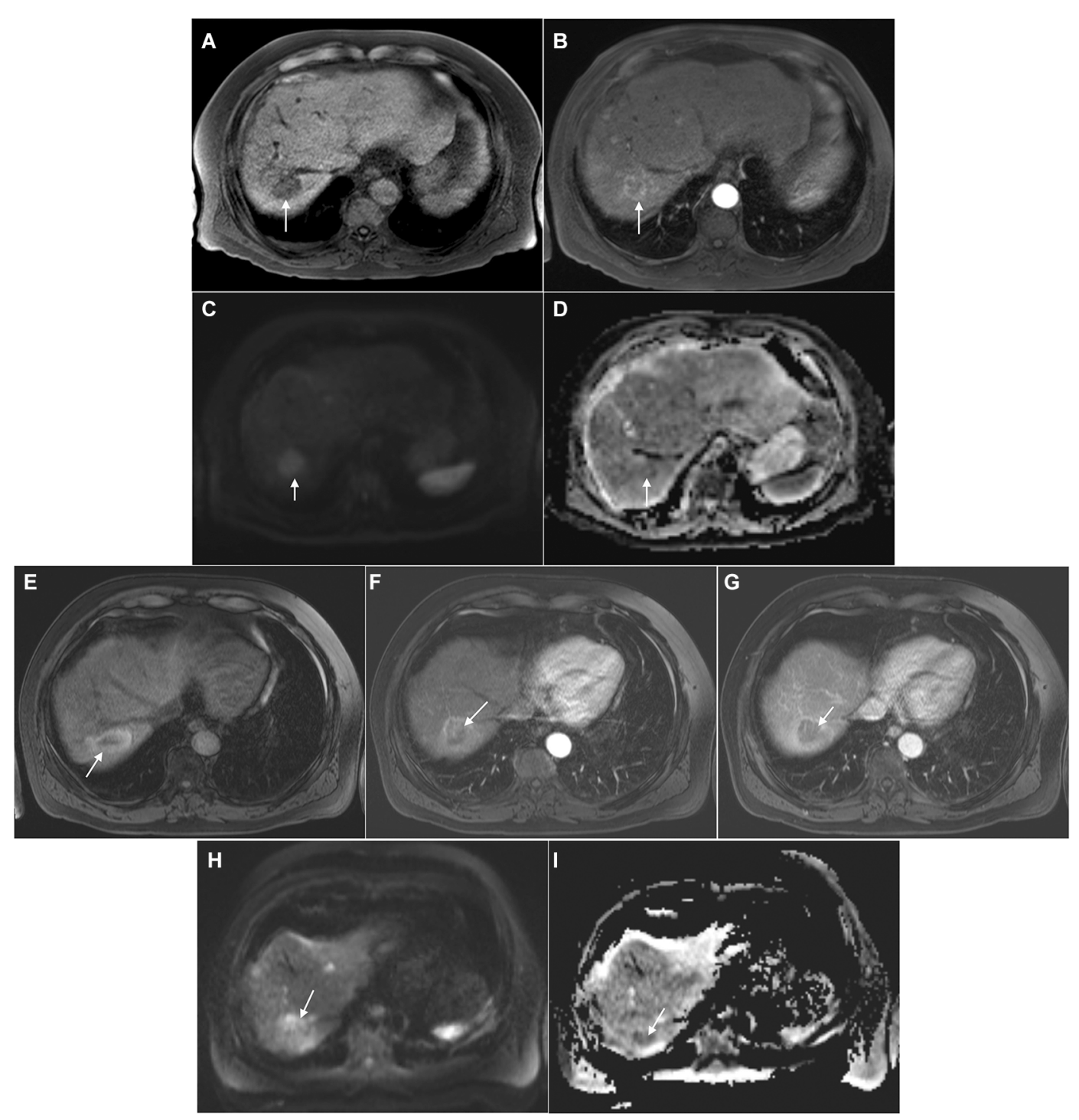

Figure 9 A 69-year-old male with a history of bladder cancer, alcoholic cirrhosis, and HCC.

Notes: Residual HCC. Pre-microwave HCC ablation imaging with the HCC tumor in segment VII (arrow) is shown in (A) TI-precontrast, (B) TI-postcontrast, (C) DWI, and (D) ADC map. Note the HCC has arterial enhancement (B) and restricted diffusion (C, D). Post-microwave HCC ablation imaging I month later is shown in (E) TI-precontrast, (F) TI-postcontrast arterial phase, (G) TI-postcontrast delay phase, (H) DWI, and (I) ADC map. Postablation shows hemorrhage with central necrosis (arrow in $\mathbf{E}$ ). There is nodular arterial enhancement with washout just superior to the ablation zone (arrow in $\mathbf{F}$ and $\mathbf{G}$ ) with restricted diffusion (arrow in $\mathbf{H}$ and $\mathbf{I}$ ). These findings are indicative of residual or recurrent HCC.

Abbreviations: HCC, hepatocellular carcinoma; DWI, diffusion-weighted imaging; ADC, apparent diffusion coefficient.

is a benign tumor containing numerous dilated vascular spaces. After contrast injection, these tumors may show the classic pattern of discontinuous nodular peripheral enhancement in the arterial phase due to slow blood flow into the vascular spaces. ${ }^{11}$ This is followed by persistent and progressive enhancement centripetally in delayed phases of imaging (Figure 5E and F). Bland versus tumor thrombosis can further be distinguished by the enhancement of tumor thrombus (Figure 10).

Gadoxetic acid (Eovist) and gadobenate dimeglumine (MultiHance) are unique among the gadolinium chelates due to their excretion by the liver. These are the so-called hepatobiliary agents. In the case of gadoxetic acid, hepatic excretion is $\sim 50 \%$, which allows imaging in the hepatobiliary phase at $\sim 20$ minutes following injection. The uptake of Gd-EOB-DTPA within the liver is a function of various transporters expressed within the sinusoids, specifically organic anion-transporting polypeptide 1B1 (OATP1B1) and/or OATP1B3. ${ }^{12-14}$ Focal nodular hyperplasia (FNH) that expresses OATP1B1/B3 demonstrates intense homogeneous enhancement in the late arterial phase and becomes isointense to the liver parenchyma. ${ }^{15}$ Hepatobiliary phase imaging with gadoxetic acid or gadobenate dimeglumine can diagnose FNH. FNHs contain functioning hepatocytes and thus take up contrast in the hepatobiliary phase of imaging. These thus appear hyperintense compared to liver parenchyma (Figure 

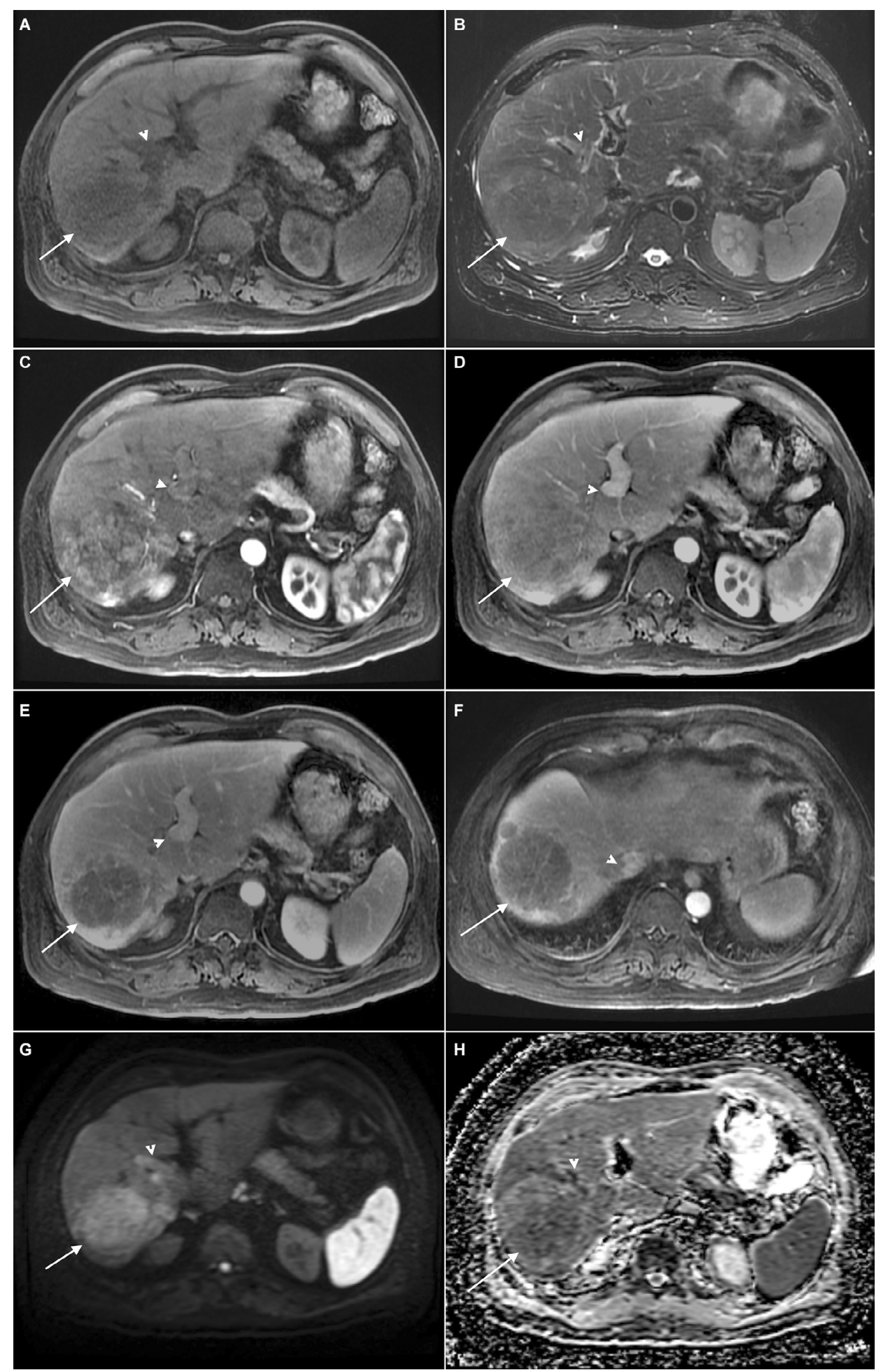

Figure 10 A 64-year-old male with history of HCC.

Notes: Tumor vein thrombosis. (A) TIW-FS precontrast; (B) T2W; (C) TI-FS-postcontrast early arterial phase; (D) TI-FS-postcontrast late arterial phase; (E) TI-FSpostcontrast portal phase; (F) TI-FS-postcontrast portal phase with axial image just superior to image (E) demonstrating tumor invading into the IVC (arrowhead). (G) DWl; (H) ADC map. A large HCC (arrow in A-H) in segment VIII is seen with hypointense TI signal (A), heterogeneous T2 signal (B), arterial enhancement (C) with washout (E), and with restricted diffusion (G, H). This HCC has invaded into the right branch of the portal vein (arrowhead A-E, G-H) causing a filling defect in the enhancing vessels (D, E). This thrombus is a tumor rather than a "bland" thrombus because it shows enhancement (C) and restricted diffusion (G, H). This tumor also invades into the right hepatic vein and extends into the IVC (arrowhead in $\mathbf{F}$ ).

Abbreviations: HCC, hepatocellular carcinoma; TIW-FS, TI-weighted-fat suppressed; DWI, diffusion-weighted imaging; ADC, apparent diffusion coefficient; IVC, inferior vena cava. 

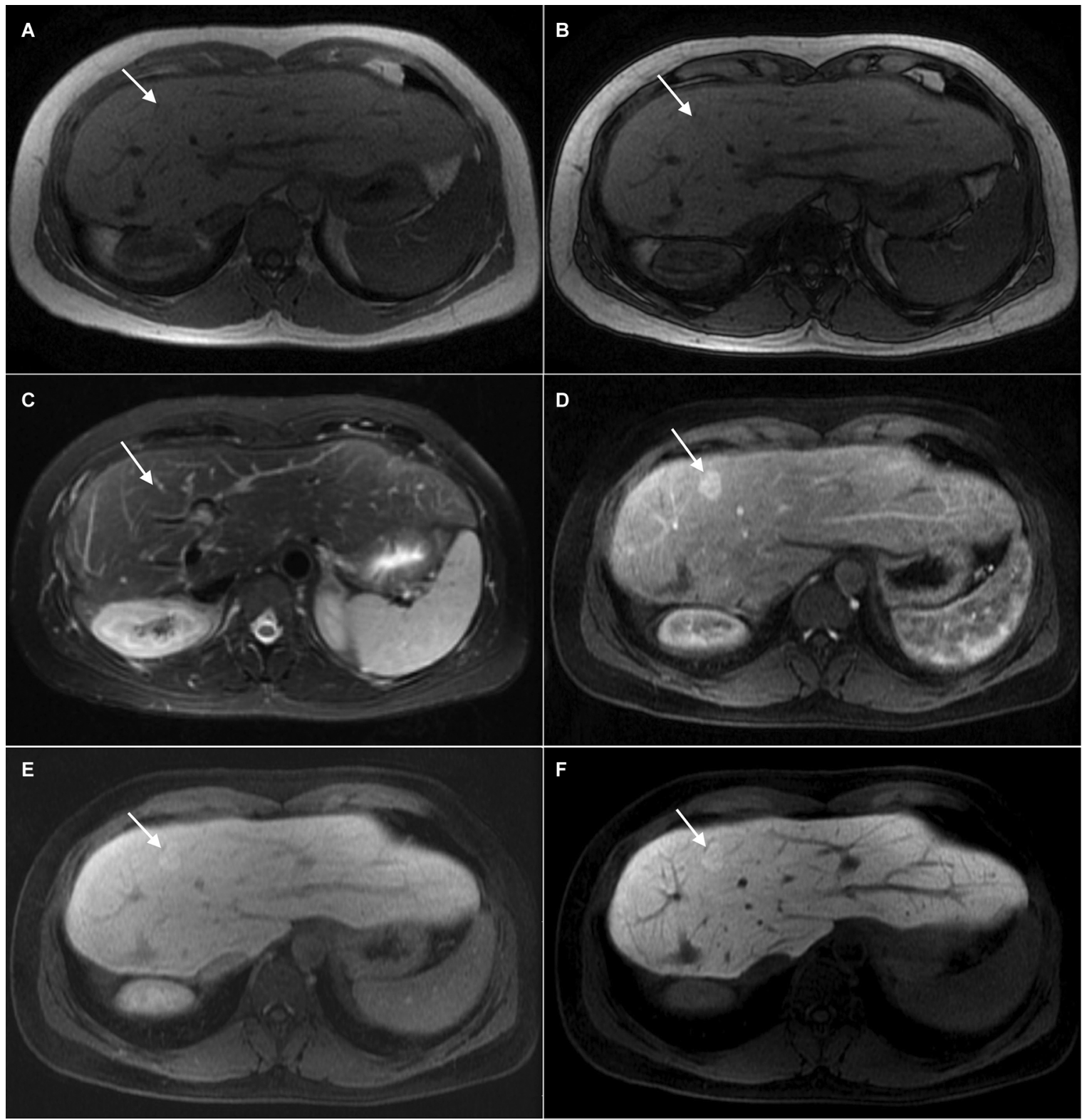

$\mathbf{F}$
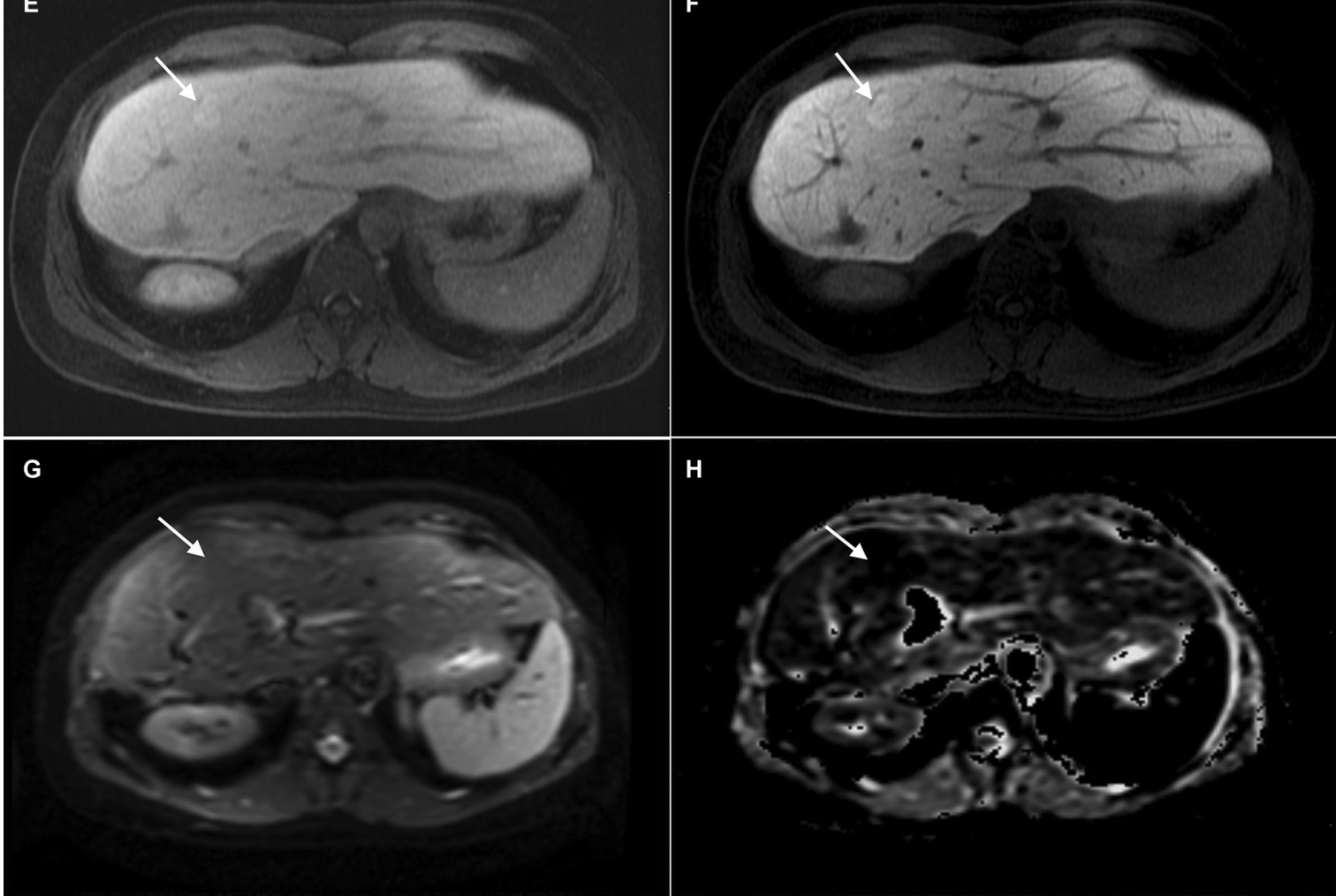

H

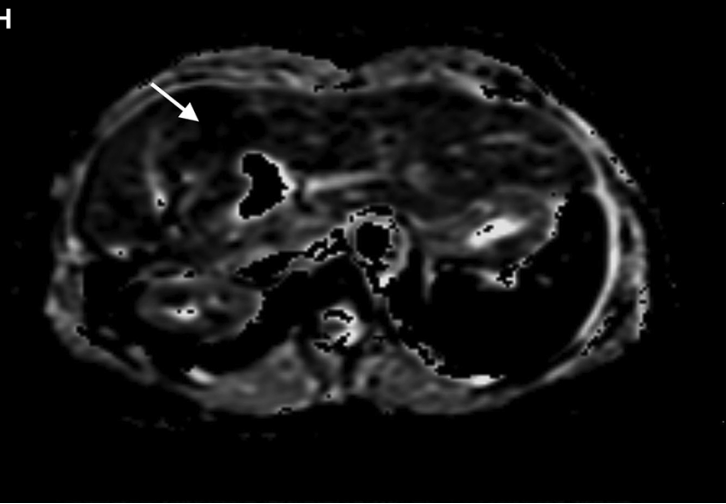

Figure II A 46-year-old female with metastatic rectal cancer to the liver.

Notes: FNH. (A) TI-in-phase; (B) TI-out-of-phase; (C) T2-FS; (D) TI-postcontrast arterial phase using gadoxetic acid. (E) TI-postcontrast 5-minute delay; (F) TIpostcontrast 20-minute delay. (G) DWI; (H) ADC map. A lesion in segment VIII (arrow A-H) shows isointense TI (A, B) and isointense T2 signal (C). This lesion shows intense arterial enhancement (D) that persists with uptake of gadoxetic acid in the delayed phase (E). Note the lesion still retains gadoxetic acid contrast after 20-minute imaging (F). This finding is classic for FNH. FNH is a benign lesion but does not show any restricted diffusion (low DWI signal) (G).

Abbreviations: $\mathrm{FNH}$, focal nodular hyperplasia; DWI, diffusion-weighted imaging; ADC, apparent diffusion coefficient. 
11). Other lesions such as cysts, metastases, hemangiomas, and hepatic adenomas (Figure 6) lack interlobular hepatic ducts and thus appear hypoenhancing relative to parenchyma in the hepatobiliary phase.

$\mathrm{HCC}$ is the most common primary hepatic malignancy and usually occurs in patients with cirrhosis. HCC is one of the few tumors that can be diagnosed with imaging to obviate the need for biopsy. The classic appearance of HCC is enhancement in the late arterial phase. In the portal venous phase, HCC is isointense to the liver. In the delayed phase, HCC is hypointense to the liver. This refers to washout. The net effect is that HCC appears to have "washed out" when moving from arterial to portal venous phases of imaging (Figure 4). ${ }^{16}$ According to the American Association for the Study of Liver Diseases, a hepatic lesion $>1 \mathrm{~cm}$ that demonstrates arterial enhancement with washout in the venous or delayed phase in MR or CT is certain enough to be HCC that histological confirmation is unnecessary. ${ }^{17,18}$ The American College of Radiology has developed a LIRADS system to categorize liver lesions for patients with cirrhosis or other risk factors for developing HCC. ${ }^{19}$ A LIRADS score of 1 or 2 is considered benign, while a score of $3-5$ is considered high probability for HCC. For hepatobliary agents, HCC are most commonly hypointense to the liver in the hepatobiliary phase. In particular, gadoxetic acid-enhanced magnetic resonance imaging, abbreviated as EOB-MRI, has recently been shown to be sensitive in distinguishing early HCC from dysplastic nodules. ${ }^{20}$ This is due to the expression of OATP1B1/B3. This distinction is more reliable in poorly differentiated HCC than in well-differentiated HCC. However, uptake of gadoxetic acid in the hepatobiliary phase has been reported on all types of HCC. They all, to a certain degree, express OATP1B1/B3.

\section{Conclusion}

This is an overview of the liver MRI. We have presented the basic sequences used in liver imaging and the added value of each for the detection and characterization of liver pathology. There are many more advanced MR sequences including MR techniques that are currently being developed in laboratory and early clinical trials which are not discussed herein. Each MR sequence is precisely engineered to probe a unique characteristic of a lesion or organ. MR is advantageous due to other modalities because of its high contrast resolution and lack of ionizing radiation. The latter feature also makes MR an important modality for noninvasive disease monitoring.

\section{Disclosure}

The authors report no conflicts of interest in this work.

\section{References}

1. Bushberg JT, Seibert AJ, Leidholdt EM, Boone JM. The Essential Physics of Medical Imaging. 2nd ed. Philadelphia, PA: Lippincott Williams \& Wilkins; 2001.

2. Pooley RA. AAPM/RSNA physics tutorial for residents: fundamental physics of MRI. Radiographics. 2005;25(4):1087-1099.

3. McEvoy SH, McCarthy CJ, Lavelle LP, et al. Hepatocellular carcinoma: illustrated guide to systematic radiologic diagnosis and staging according to guidelines of the American Association for the Study of Liver Diseases. Radiographics. 2013;33(6):1653-1668.

4. Matos AP, Velloni F, Ramalho M, AlObaidy M, Rajapaksha A, Semelka RC. Focal liver lesions: practical magnetic resonance imaging approach World J Hepatol. 2015;7(16):1987-2008.

5. Prasad SR, Wang H, Rosas H, et al. Fat-containing lesions of the liver: radiologic-pathologic correlation. Radiographics. 2005;25(2):321-331.

6. Pokharel SS, Macura KJ, Kamel IR, Zaheer A. Current MRI lipid detection techniques for diagnosis of lesions in the abdomen and pelvis. Radiographics. 2013;33(3):681-702.

7. Chavhan GB, Babyn PS, Thomas B, Shroff MM, Haacke EM. Principles, techniques, and applications of T2*-based MRI and its special applications. Radiographics. 2009;29:1433-1449.

8. Kele PG, van der Jagt EJ. Diffusion weighted imaging in the liver. World $J$ Gastroenterol. 2010;16(13):1567-1576.

9. Koh DM, Collins DJ. Diffusion-weighted MRI in the body: applications and challenges in oncology. AJR Am J Roentgenol. 2007;188(6): $1622-1635$.

10. Vallejo Desviat P, Martinez De Vega V, Recio Rodriguez M, Jimenez De La Pena M, Carrascoso Arranz J. Diffusion MRI in the study of hepatic lesions. Cir Esp. 2013;91(1):9-16.

11. Goodwin MD, Dobson JE, Sirlin CB, Lim BG, Stella DL. Diagnostic challenges and pitfalls in MRI with hepatocyte-specific contrast agents Radiographics. 2011;31(6):1547-1568.

12. Tsuboyama T, Onishi H, Kim T, et al. Hepatocellular carcinoma: hepatocyte-selective enhancement at gadoxetic acid-enhanced MRI correlation with expression of sinusoidal and canalicular transporters and bile accumulation. Radiology. 2010;255(3):824-833.

13. Haimerl M, Verloh N, Zeman F, et al. Gd-EOB-DTPA-enhanced MRI for evaluation of liver function: comparison between signal-intensitybased indices and T1 relaxometry. Sci Rep. 2017;7:43347.

14. Van Montfoort JE, Stieger B, Meijer DK, Weinmann HJ, Meier PJ, Fattinger KE. Hepatic uptake of the magnetic resonance imaging contrast agent gadoxetate by the organic anion transporting polypeptide Oatp1. J Pharmacol Exp Ther. 1999;290(1):153-157.

15. Seale MK, Catalano OA, Saini S, Hahn PF, Sahani DV. Hepatobiliaryspecific MR contrast agents: role in imaging the liver and biliary tree. Radiographics. 2009;29(6):1725-1748.

16. Ishigami K, Yoshimitsu K, Nishihara Y, et al. Hepatocellular carcinoma with a pseudocapsule on gadolinium-enhanced MR images: correlation with histopathologic findings. Radiology. 2009;250(2):435-443.

17. Bruix J, Sherman M, Practice Guidelines Committee AASLD. Management of hepatocellular carcinoma. Hepatology. 2005;42(5): 1208-1236.

18. Bruix J, Sherman M, American Association for the Study of Liver Diseases (AASLD) Practice Guidelines. Management of hepatocellular carcinoma: an update. Hepatology. 2011;53(3):1020-1022.

19. American College of Radiology. Liver Imaging Reporting and Data System; 2017. Available from: https://www.acr.org/Quality-Safety/ Resources/LIRADS. Accessed September 17, 2017.

20. Ichikawa T, Sano K, Morisaka H. Diagnosis of pathologically early HCC with EOB-MRI: experiences and current consensus. Liver Cancer 2014;3:97-107. 


\section{Publish your work in this journal}

The Journal of Hepatocellular Carcinoma is an international, peerreviewed, open access journal that offers a platform for the dissemination and study of clinical, translational and basic research findings in this rapidly developing field. Development in areas including, but not limited to, epidemiology, vaccination, hepatitis therapy, pathology and molecular tumor classification and prognostication are all considered for publication. The manuscript management system is completely online and includes a very quick and fair peer-review system, which is all easy to use. Visit http://www.dovepress.com/testimonials.php to read real quotes from published authors.

Submit your manuscript here: https://www.dovepress.com/journal-of-hepatocellular-carcinoma-journal 